\title{
Incidencia de la antropometría, práctica de actividad física, estilos de aprendizaje, motivos, actitudes y estrategias de aprendizaje sobre el rendimiento académico de estudiantes de Santiago de Chile
}

Incidence of anthropometry, physical activity practice, learning styles, motives, attitudes, and learning strategies on the academic performance of students from Santiago of Chile

*Fernando Maureira Cid, *Elvira Palma Gajardo, *Roberto Medina Saavedra, *Álvaro Segueida Lorca, *Luis Valenzuela

Contreras, ${ }^{* *}$ Elizabeth Flores Ferro

*Universidad Católica Silva Henríquez (Chile), **Universidad Bernardo O’Higgins (Chile)

\begin{abstract}
Resumen. Existen variados factores que influyen en el rendimiento académico como elementos psicosociales, socioeconómicos, familiares, años de programas de estudio, las metodologías de enseñanza, coeficiente Intelectual, motivación, etc. El objetivo del presente estudio fue conocer la incidencia de la práctica de ejercicio físico, composición corporal, estilos de aprendizaje, motivación y hábitos de estudio sobre el rendimiento académico. La muestra estuvo constituida por 713 estudiantes varones de seis colegios de Santiago de Chile que cursaban séptimo y octavo de primaria, y primero y segundo de secundaria. Se aplicó el cuestionario de Honey-Alonso en su versión abreviada (CHAEA-36), el inventario de motivos actitudes y estrategias para el aprendizaje, la adaptación de la encuesta nacional de hábitos de actividad física y deporte en la población chilena, se calculó el IMC, se midió el pliegue tricipital y la circunferencia de cintura, y se recopilo el rendimiento académico del primer semestre 2018. Los resultados muestran correlaciones bajas y negativas entre el estilo activo y todos los promedios de notas, en tanto, el estilo reflexivo se correlaciona de manera positiva pero leve con todos los promedios. Los puntajes de la sub-escala metas, condiciones de estudio e implicaciones en el estudio se relacionan de forma positiva y leve con todas las notas obtenidas. Finalmente, las variables antropométricas peso, estatura, perímetro de cintura y la práctica de actividad física son responsables de menos del $1 \%$ de las variaciones de las notas de los estudiantes de la muestra.
\end{abstract}

Palabras claves: rendimiento académico, antropometría, ejercicio físico, estilos de aprendizaje, motivación, estrategias de aprendizaje.

Abstract. There exist several factors that influence academic performance, among them psychosocial and socioeconomic elements, family, study program duration, pedagogical methodologies, intellectual coefficient, motivation, etc. The aim of the present study was to evaluate the incidence of physical exercise practice, corporal composition, learning styles, motivation, and study habits on academic performance. The sample was constituted by 713 male students from six colleges of Santiago of Chile, enrolled in the seventh and eighth grade of primary education, and in the first and second of secondary. The short version of the Honey-Alonso's questionnaire (CHAEA36) was applied, alongside with the inventory of motives, attitudes, and strategies for learning, an adaptation of the national survey of habits of physical activity and sport in the Chilean population, IMC calculations, skinfold measurements on the triceps, and waist circumference. In addition, academic performance from the first semester of 2018 was consulted. The results showed low and negative correlations between active style and all averages grades. However, reflexive style was slightly but positively correlated with all grade averages. The scores from the sub-scale goals, conditions of study, and implications in the study slightly and positively related with all grades. Finally, the anthropometric variables of weight, height, waist circumference, as well as physical activity practice are responsible for less than $1 \%$ of the variations of grades of the students in our sample.

Keywords: academic performance, anthropometry, physical exercise, learning styles, motivation, learning strategies.

\section{Introducción}

La evaluación del aprendizaje y la mejora de este, son dos pilares fundamentales en el proceso educativo, convirtiéndose en las últimas décadas casi en el único elemento valorado en los sistemas formativos de occidente (Edel, 2003). El análisis del aprendizaje se ha restringido a la valoración del rendimiento académico, que para Jiménez (2000) corresponde al nivel de conocimiento que un estudiante demuestra en una determinada disciplina en relación con otros estudiantes de su edad y nivel académico. Para Tonconi (2010) el rendimiento académico es el nivel de conocimiento sobre un área evidenciado con indicadores cuantitativos y expresados en calificaciones ponderadas en el sistema vigesimal. Pizarro y Clark (1998) sostienen que el rendimiento académico es la medida de capacidad de respuesta del individuo, la cual se expresa en forma estimativa cuando la persona ha aprendido a partir del resultado de un proceso de instruc-

Fecha recepción: 23-01-19. Fecha de aceptación: 02-06-19 Fernando Maureira Cid maureirafernando@yahoo.es ción o formación. Finalmente, Aguirre, Castro, y Adasme (2009) plantean que el rendimiento académico es lo que se denomina como la calificación escolar, indicador de logro escolar individual, que se observa a través de las evaluaciones del alumno dejando ver el nivel de conocimientos académicos adquiridos en un determinado momento del proceso educativo.

Existen variados factores que influyen en el rendimiento académico como elementos psicosociales, socioeconómicos, familiares, años de programas de estudio, las metodologías de enseñanza (Benítez, Giménez, y Osicka, 2000; Cano, 2008; González, 2015), coeficiente Intelectual (Aravena, Maureira, Flores y González, 2017; Kohler, 2013; Pérez y Castejon, 2006), estilos de aprendizaje (Acevedo y Martínez, 2011; Gallego y Nevot, 2007; Juárez, Rodríguez y Escoto, 2012; Maureira, Bahamondes y Aravena, 2015; Quintanal, 2011), práctica de ejercicio físico (Haapala, Väistö, Lintu, Westgate, Ekelund, Poikkeus, et al., 2017; Kao, Westfall, Parks, Pontifex y Hillman, 2017; Maureira, Díaz, Foss, Ibañez, Molina, Aravena, et al., 2014; Owen, Parker, Astell y Lonsdale, 2018), motivación (Antolin, 2013; Chocontá, 2016; Maquilón y Hernández, 2011), etc., convirtiéndose el desempeño académico en un 
fenómeno multifactorial con componentes biológicoscognitivos y socio-culturales.

El término estilo de aprendizaje se refiere al hecho de que cada persona utiliza su propio método para aprender, aunque las estrategias varían según lo que se quiera conocer (Cazau, 2004). Para Keefe (1988) los estilos de aprendizaje son modos fisiológicos, cognitivos y emocionales de como el ser humano recibe y procesa la información. Para Alonso, Gallego y Honey (1994:104) «los estilos de aprendizaje son los rasgos cognitivos, afectivos y fisiológicos que sirven como indicadores relativamente estables, de cómo los alumnos perciben interacciones y responden a sus ambientes de aprendizaje». Hernández y Hervás (2005) afirman que la identificación de los estilos de aprendizaje sirve para diseñar estrategias de enseñanza según los perfiles de los alumnos. Para Ossa y Lagos (2013) el hecho de conocer los estilos de aprendizaje de los estudiantes puede ayudar a constituir ambientes propicios para un aprendizaje más eficaz.

En la actualidad existen diversos trabajos que relacionan los estilos de aprendizaje con el rendimiento académico (Álvarez y Albuerne, 2001; Blumen, Rivero y Guerrero, 2011; Camarero, Martín del Buey y Herrero, 2000; González y González, 2005; Montero, Sepúlveda y Contreras, 2011; Ruiz, Trillos y Morales, 2006; Serra-Olivares, Muñoz, Cejudo \& Gil, 2017; Suazo, 2007; etc.).

Por otra parte, Pintrich y Schunk (2006) definen la motivación como el proceso que nos dirige hacia el objetivo o la meta de una actividad, que la influye y mantiene. Por lo tanto, es más un proceso que un producto, implica la existencia de unas metas, requiere cierta actividad (física o mental), por lo que se considera una actividad decidida y sostenida. En relación con el concepto de hábitos de estudio Belaunde (1994) afirma que se refiere a la forma en que el individuo se enfrenta cotidianamente al quehacer académico. Esto se refiere a tener la costumbre de aprender permanentemente, lo cual implica la forma en que el individuo organiza su tiempo, el espacio, las técnicas y los métodos necesarios para estudiar. Cartagena (2008) desarrolla un enfoque similar, al plantear que el hábito de estudio es el método que el estudiante emplea para asimilar el aprendizaje, evitar las distracciones, enfocar su atención hacia la materia estudiada y el esfuerzo que realiza durante el proceso de desarrollar las tareas. El autor afirma que los hábitos se crean por la repetición y acumulación de actos, mientras mayor sea la frecuencia de estudiar en un mismo horario y lugar, mayor será el hábito de estudiar.

En los últimos años, han surgido nuevos factores que representan importantes elementos dentro del contexto académico, siendo uno de los más revolucionarios el ejercicio físico. Esto ha sido comúnmente asociado a la disminución de enfermedades crónicas no transmisibles como la diabetes, las enfermedades cardiovasculares y las enfermedades respiratorias, (Gami, Witt, Howard, Erwin, Gami, Somers, et al., 2007; Romero, 2006), además de la mejora de condición física, la coordinación y las cualidades motrices (Wilmore y Costill, 2007). Pero el ejercicio físico también ha sido vinculado a mejoras en estructuras cerebrales, lo cual conlleva a una mejora de funciones cognitivas como la atención, memoria, planificación, inhibición, etc. (Maureira, 2018). Incluso se ha establecido una relación entre la práctica de ejercicio físico y el rendimiento académico (Castelli, Hillman, Buck y Erwin, 2007; Ishihara, Morita, Nakajima, Okita, Yamatsu y Sagawa, 2018; Maureira, Bravo, Aguilera, Bahamondes \& Véliz, 2019; Oliveira, Pizarro, Costa, Fernandes, Silva, Mota, et al., 2017; Prieto y Martínez, 2016; Reed, Einstein, Hahn, Hooker, Gross y Kravitz, 2010, etc.).

En base a todos los antecedentes mencionados es que surge la presente investigación, con el propósito de indagar en la incidencia de la práctica de ejercicio físico, composición corporal, estilos de aprendizaje, motivación y hábitos de estudio sobre el rendimiento académico de estudiantes de Santiago de Chile. La hipótesis de trabajo sugiere que las variables estudiadas inciden positivamente en el rendimiento académico de la muestra.

\section{Metodología}

\section{Muestra}

Fue de tipo no probabilística intencionada. Estuvo constituida por 713 estudiantes varones de seis colegios de Santiago de Chile. Del total, 169 sujetos (23,7\%) cursaban séptimo de primaria, 182 (25,5\%) octavo de primaria, 184 (25,8\%) primero de secundaria y 178 (25\%) segundo de secundaria. La edad mínima fue de 12 años y la máxima de 17, con una media de 14,1 $\pm 1,2$. Esta investigación fue aprobada por el comité de ética de la Universidad Católica Silva Henríquez mediante el acta s/n de marzo de 2018. Todos los participantes y sus apoderados firmaron un consentimiento informado.

\section{Instrumentos}

Cuestionario de Honey-Alonso en su versión abreviada (CHAEA-36): instrumento que consta de 36 ítems de respuesta dicotómica (Si-No), agrupadas en 9 ítems para cada estilo de aprendizaje (activo, reflexivo, teórico y pragmático). Para cada sub-escala se obtiene una puntuación sumando el número de respuestas positivas. Este cuestionario corresponde a una adaptación del Cuestionario Honey-Alonso de Estilos de Aprendizaje (CHAEA) con 80 ítems (Alonso, 1991). El CHAEA-36 fue validado en estudiantes de enseñanza media de Chile por Aravena, Maureira, Flores, Lourido y Véliz (2017).

Inventario de motivos actitudes y estrategias para el aprendizaje: instrumento que consta de 62 ítems tipo Likert con opciones de 1 a 5 . El inventario se divide en 6 subescalas: metas (con 19 ítems), actitud ante la tarea (con 6 ítems), motivos personales (con 15 ítems), condiciones de estudio (con 6 ítems), estrategias de aprendizaje (con 9 ítems), implicaciones en el estudio (con 7 ítems). Para cada subescala se obtiene una puntuación equivalente a la media de los puntajes de sus ítems. Este instrumento fue desarrollado por Bernstein (1997) y validado por Boza y Toscano (2012). En Chile el inventario fue validado en estudiantes de enseñanza media por Aravena, Maureira, Flores y González (2017).

Adaptación de la encuesta nacional de hábitos de actividad física y deporte en la población chilena: se utilizaron las preguntas más acordes a estudiantes escolares de la encuesta nacional del año 2016. Con esto se obtuvo la información sobre la práctica de actividad física y actividad deportiva que desarrolla la muestra. El instrumento fue validado en 
Chile por el Ministerio de Deporte (2016).

Seutilizó una balanza mecánica SECA 700 con un tallímetro telescópico SECA 220, con precisión de 50 gramos y un centímetro respectivamente. Para la evaluación del pliegue tricipital y de la circunferencia de cintura se utilizó el adipómetro y la cinta métrica del kit antropométrico ROSSCRAFT.

Rendimiento académico: se obtuvo a través de la recopilación de las actas de notas finales del primer semestre 2018. Se utilizaron las notas de lenguaje, matemáticas, ciencias e historia.

\section{Procedimiento}

La recolección de datos tuvo una duración 60-70 minutos aproximadamente y fue realizada en forma grupal durante las horas de clases. Se aplicaron el CHAEA-36, el Inventario de motivos actitudes y estrategias para el aprendizaje y la encuesta de hábitos de actividad física y deporte. Posteriormente, en forma individual se obtuvieron los datos de talla, peso, circunferencia de cintura y pliegue del tríceps. El evaluador fue un profesor de educación física con vasta experiencia en mediciones antropométricas y con la certificación internacional de cineantropometría nivel 3 de la International Society for the Advancement of Kinanthropometry (ISAK). Las actas con el rendimiento académico del primer semestre 2018 de cada estudiante se solicitaron al establecimiento educacional respectivo.

\section{Análisis de datos}

Se utilizó el programa estadístico SPSS 24.0 para Windows. Para conocer los estilos de aprendizaje, los motivos, actitudes y estrategias para el aprendizaje, la práctica de actividad física, la composición corporal y el rendimiento académico de la muestra se aplicarán análisis descriptivos como medias y desviaciones estándar. Para determinar la normalidad de los datos se utilizaron pruebas de Kolmogorov-Smirnov (KS). También se aplicaron análisis inferenciales como ANOVA para comparar todas las variables según edad de la muestra, análisis de correlaciones de Pearson para conocer la posible relación de los estilos de aprendizaje, motivos, actitudes y estrategias para el aprendizaje, practica de actividad física y composición corporal con el rendimiento académico de la muestra y en caso de ser necesario se aplicarán análisis de regresiones múltiples para determinar la incidencia de las variables correlacionas con las notas obtenidas por la muestra durante el primer semestre 2018. Se utilizó un nivel de significancia de $\mathrm{p}<.05$.

\section{Resultados}

La prueba de Kolmogorov-Smirnov (KS) entregó valores $\mathrm{p}>.05$ para todas las variables numéricas estudiadas, razón por la cual se procedió a utilizar estadística paramétrica. Las medias y desviaciones estándar de estas variables se presentan en la tabla 1 . También se aprecian los valores p de las pruebas ANOVA comparando los resultados por cursos. En las variables antropométricas, como era de esperarse, existen diferencias significativas en el peso y la estatura, ambas variables aumentan con la edad y, por ende, con el aumento del nivel escolar (curso). En forma opuesta, el pliegue del tríceps disminuye con los cursos $(\mathrm{p}=.000)$. En relación con los estilos de aprendizaje sobre se observan diferencias en el estilo reflexivo, donde $1^{\circ}$ y $2^{\circ}$ de secundaria obtuvieron medias mayores a $7^{\circ}$ y $8^{\circ}$ de primaria $(p=0,000)$. En las puntuaciones de las subescalas del cuestionario de motivos, actitudes y estrategias de aprendizaje no se aprecian diferencias significativas entre los cursos. En el ámbito de los promedios de notas, sólo ciencia presenta diferencias entre los cursos, siendo $1^{\circ}$ y $2^{\circ}$ de secundaria lo que obtienen promedios más altos $(\mathrm{p}=.000)$.

En la tabla 2 se observan los porcentajes de tiempo de practica de actividad física de la muestra. También se muestran los valores p de las pruebas de chi-cuadrado comparando los resultados de las categorías por cursos. Solo se aprecian diferencias significativas en la frecuencia de practica semanal, donde $1^{\circ}$ y $2^{\circ}$ de secundaria realizan actividad física con más frecuencia que $7^{\circ}$ y $8^{\circ}$ de primaria $(\mathrm{p}=.011)$.

\begin{tabular}{|c|c|c|c|c|c|}
\hline & $7^{\circ}$ & $8^{\circ}$ & $1^{\circ}$ & $2^{\circ}$ & $p$ \\
\hline \multicolumn{6}{|l|}{ Antropometría } \\
\hline Peso (kilos) & $55,9 \pm 3,3$ & $59,2 \pm 12,1$ & $63,2 \pm 2,7$ & $66,0 \# 2,3$ & $.000^{* *}$ \\
\hline Estatura (metros) & $1,56 \pm 0,08$ & $1,63 \pm 0,08$ & $1,67 \pm, 14$ & $1,70 \pm, 07$ & $.000 * *$ \\
\hline IMC & $22,7 \pm 4,14$ & $22,2 \pm 3,75$ & $22,1 \pm 4,08$ & $22,9 \pm 3,74$ & .205 \\
\hline Pliegue tríceps (cm) & $14,1 \pm 5,19$ & $12,1 \pm 4,77$ & $11,4 \pm 4,65$ & $10,7 \sharp 4,31$ & $.000 * *$ \\
\hline Cintura $(\mathrm{cm})$ & $74,3 \pm 9,93$ & $74,4 \pm 8,58$ & $74,7 \pm 8,94$ & $76,1 \pm 8,57$ & .204 \\
\hline \multicolumn{6}{|l|}{ Estilos de aprendizaje } \\
\hline Activo & $5,27 \pm, 86$ & $5,31 \pm$ ㄱ, 73 & $5,12 \pm 2,00$ & $5,19 \#, 98$ & .780 \\
\hline Reflexivo & $5,88 \pm, 91$ & $5,39 \pm, 97$ & $6,10 \pm, 86$ & $6,25 \#, 84$ & $.000^{* *}$ \\
\hline Teórico & $6,27 \pm, 69$ & $5,87 \#, 79$ & $6,12 \#, 77$ & $6,08 \#, 63$ & .187 \\
\hline Pragmático & $5,89 \pm, 67$ & $5,66 \boxplus, 74$ & $5,99 \#, 56$ & $6,10 \#, 64$ & .073 \\
\hline \multicolumn{6}{|l|}{$\begin{array}{l}\text { Motivos, actitudes y } \\
\text { estrategias de aprendizaje }\end{array}$} \\
\hline Metas & $3,15 \pm 0,71$ & $3,03 \pm 0,68$ & $3,04 \pm n, 63$ & $3,13 \pm, 63$ & .238 \\
\hline Actitud ante la tarea & $3,37 \pm 0,77$ & $3,36 \pm 0,58$ & $3,33 \pm n, 52$ & $3,43 \pm 0,51$ & .506 \\
\hline Motivos personales & $3,73 \pm, 76$ & $3,63 \pm 0,68$ & $3,62 \pm 0,59$ & $3,70 \pm 0,55$ & .255 \\
\hline Condiciones de estudio & $3,27 \pm 0,86$ & $3,13 \pm 0,83$ & $3,12 \pm n, 64$ & $3,12 \pm, 75$ & .235 \\
\hline Estrategias de aprendizaje & $3,28 \pm, 00$ & $3,21 \pm 7,88$ & $3,23 \pm n, 76$ & $3,32 \pm, 78$ & .621 \\
\hline Implicaciones en el estudio & $3,48 \pm 0,94$ & $3,50 \pm n, 81$ & $3,59 \pm 0,61$ & $3,58 \pm 0,67$ & .398 \\
\hline \multicolumn{6}{|l|}{ Promedios de notas } \\
\hline Lenguaje & $5,1 \pm 0,84$ & $5,1 \pm, 69$ & $5,1 \pm, 95$ & $5,1 \pm 0,73$ & .800 \\
\hline Matemáticas & 4,9\#0,88 & 4,9 & $5,0 \#, 17$ & $5,0 \pm 0,99$ & .602 \\
\hline Historia & $5,2 \pm 0,81$ & $5,1 \pm, 78$ & $5,0 \pm, 89$ & $5,1 \pm 0,80$ & .176 \\
\hline Ciencias & $5,0 \pm 0,85$ & $5,0 \pm 0,83$ & $5,3 \pm, 75$ & $5,2 \pm 0,72$ & $.000^{* *}$ \\
\hline
\end{tabular}

**Diferencia significativa al nivel .01

\begin{tabular}{|c|c|c|c|c|c|}
\hline & $7^{\circ}$ & $8^{\circ}$ & $1^{\circ}$ & $2^{\circ}$ & $p$ \\
\hline Practico & $134(79,3 \%)$ & $137(75,3 \%)$ & $146(79,3 \%)$ & $130(73,0 \%)$ & .407 \\
\hline No practico & $35(20,7 \%)$ & $45(24,7 \%)$ & $38(20,7 \%)$ & $48(27,0 \%)$ & \\
\hline Todos los días & $10(6,8 \%)$ & $4(3,1 \%)$ & $26(19,4 \%)$ & $17(12,4 \%)$ & $.011^{*}$ \\
\hline 6 días por semana & $12(8,2 \%)$ & $15(11,5 \%)$ & $7(5,2 \%)$ & $10(7,3 \%)$ & \\
\hline 5 días por semana & $16(11,0 \%)$ & $13(10,0 \%)$ & $19(14,2 \%)$ & $17(12,4 \%)$ & \\
\hline 4 días por semana & $23(15,8 \%)$ & $31(23,8 \%)$ & $22(16,4 \%)$ & $24(17,5 \%)$ & \\
\hline 3 días por semana & $42(28,8 \%)$ & $31(23,8 \%)$ & $27(20,1 \%)$ & $35(25,5 \%)$ & \\
\hline 2 días por semana & $33(22,6 \%)$ & $19(14,6 \%)$ & $18(13,4 \%)$ & $20(14,6 \%)$ & \\
\hline 1 día por semana & $9(6,2 \%)$ & $10(7,7 \%)$ & $12(9,0 \%)$ & $8(5,8 \%)$ & \\
\hline$<1$ vez por semana & $1(0,7 \%)$ & $7(5,4 \%)$ & $3(2,2 \%)$ & $6(4,4 \%)$ & \\
\hline 30 minutos & $17(11,6 \%)$ & $19(14,1 \%)$ & $19(14,1 \%)$ & $16(11,7 \%)$ & .063 \\
\hline 45 minutos & $13(8,9 \%)$ & $15(11,5 \%)$ & $10(7,5 \%)$ & $9(6,6 \%)$ & \\
\hline 60 minutos & $24(16,4 \%)$ & $22(16,9 \%)$ & $22(16,4 \%)$ & $28(20,4 \%)$ & \\
\hline 75 minutos & $9(6,2 \%)$ & $10(7,7 \%)$ & $4(3,0 \%)$ & $8(5,8 \%)$ & \\
\hline 90 minutos & $30(20,5 \%)$ & $32(24,6 \%)$ & $21(15,7 \%)$ & $30(21,9 \%)$ & \\
\hline 120 minutos & $32(21,9 \%)$ & $22(16,9 \%)$ & $20(14,9 \%)$ & $23(16,8 \%)$ & \\
\hline$>120$ minutos & $21(14,4 \%)$ & $16(12,3 \%)$ & $38(28,4 \%)$ & $23(16,8 \%)$ & \\
\hline
\end{tabular}

En la tabla 3 se aprecian las correlaciones de Pearson entre los estilos de aprendizaje, motivos, actitudes y estrategias de aprendizaje, variables antropométricas y rendimiento académico. Además, se observan las correlaciones de Spearman entre la práctica de actividad física y los promedios obtenidos por los estudiantes de la muestra. Las variables antropométricas peso, estatura y perímetro de cintura se relacionan sólo con las notas de historia, pero explican menos del 1\% de las variaciones de los promedios académicos. Una situación similar ocurre con la práctica de actividad física, las cuales son responsables de menos del 1\% de las 
variaciones de las notas de los estudiantes de la muestra.

Se observan correlaciones bajas y negativas entre el estilo activo y todos los promedios de notas, sin embargo, los coeficientes de determinación $\left(\mathrm{r}^{2}\right)$ no superan el 3\%. El estilo reflexivo se correlaciona de manera positiva pero leve con todos los promedios, pero al igual que el caso anterior, el $\mathrm{r}^{2}$ no supera el 3\%.

Los puntajes de la sub-escala metas, condiciones de estudio e implicaciones en el estudio se relacionan de forma positiva y leve con todas las notas obtenidas, con un $\mathrm{r}^{2}$ que varía entre $1 \%$ y $8 \%$. La sub-escala motivos personales se relaciona con las notas de historia y ciencias, y la sub-escala estrategias de aprendizaje se relaciona con los promedios de lenguaje, historia y ciencias.

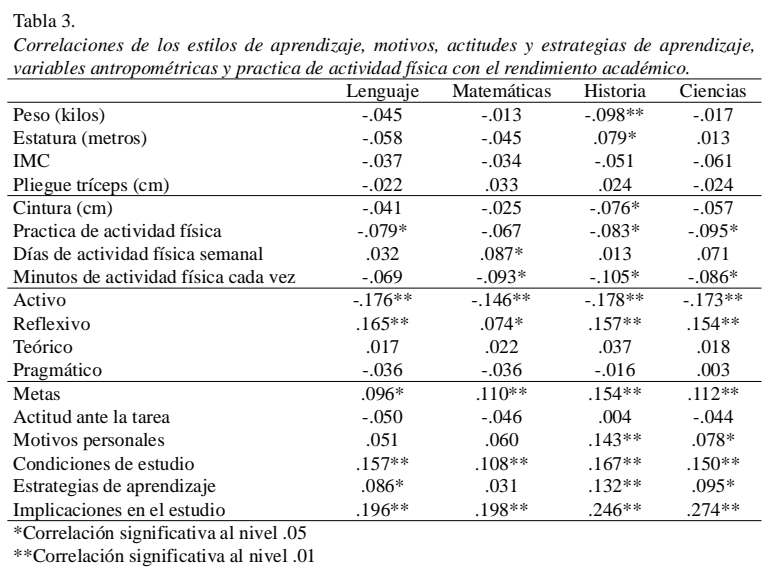

\section{Discusiones}

El objetivo de la presente investigación fue conocer la incidencia de la práctica de ejercicio físico, composición corporal, estilos de aprendizaje, motivación y hábitos de estudio sobre el rendimiento académico de los estudiantes de Santiago de Chile. Los resultados muestran que en varones las variables como el peso y estatura aumentan con la edad y los niveles de grasa en el tríceps disminuyen, lo que es coherente con la literatura (Giraldo, Uscátegui, Restrepo \& Estrada, 2012; Pinel, Chacón, Castro, Espejo, Zurita \& Pérez, 2017; Tobarra, Castro \& Badilla, 2015). En relación con la práctica de ejercicio físico, este aumenta con la edad de la muestra, lo que podría ser explicado por la necesidad de competir que presentan los escolares varones en edades de 15 o más años (Palou, Ponseti, Gili, Borras \& Vidal, 2005).

A diferencia de variadas investigaciones que relacionan positivamente la antropometría y/o la práctica de ejercicio físico con el rendimiento académico (Godoy, Valdés, Fariña, Cárcamo, Medina, Meneses, et al., 2015; Haapala, Lintu, Eloranta, Venäläinen, Poikkeus, Ahonen, et al., 2018; Kao, et al. 2017; Pellicer, García, Morales, Serra, Solana, González, et al., 2015; Van Dijk, De Groot, Savelberg, VanAcker y Kirschner, 2014; etc.) en el presente estudio se encontraron correlaciones negativas entre la práctica y el tiempo de realización de ejercicio físico con las notas obtenidas en lenguaje, matemáticas, historia y ciencias. Si bien, los coeficientes de determinación son muy bajos (menos del 1\%), es posible que la relación negativa se deba a que los estudiantes que realicen más actividad física dediquen menos tiempos a estudiar y realizar actividades escolares.
Los estudiantes de séptimo y octavo de primaria poseen un estilo de aprendizaje predominantemente teórico. Esto sujetos se caracterizan porque adaptan e integran teorías, son lógicos, sistemático, metódicos, objetivos y no gustan de la intuición y las emociones (Honey y Munford, 1992). En primero de secundaria los estudiantes son tanto teóricos como reflexivos. Esto últimos se caracterizan porque gustan de comprender significado y describir procesos, les gustan los análisis, observan desde diferentes perspectivas, observan y escuchan antes de hablar, etc. (Honey y Munford, 1992). Los estudiantes de segundo de secundaria son preferentemente reflexivos.

De los estilos de aprendizaje, el activo se relaciona de manera inversa con el rendimiento académico en lenguaje, matemáticas, historia y ciencias. Esto podría estar dado ya que los estudiantes activos son originales, creativos, actúan primero y piensan después, y les desagradan las tareas administrativas y los trabajos largo (Honey y Munford, 1992). Por su parte, el estilo reflexivo se relaciona positivamente con las notas obtenidas en la muestra. Pese a estas correlaciones, los coeficientes de determinación no superan el 3\%, dando cuenta de una influencia muy escasa de los estilos activo y reflexivo sobre las notas obtenidas por la muestra.

En relación con los motivos, actitudes y estrategias de aprendizaje las medias de todas las sub-escalas superan los 3 puntos, siendo los motivos personales lo que presentan puntuaciones más altas (trabajo, mercado, expectativas personales, profesión, responsabilidad, conocimiento, superación, vida ordenada, etc.) y las metas, las que poseen el puntaje más bajo (ser competentes en su tarea, ganar dinero, tener éxito en la vida, integrarse en la sociedad, responsabilidad social, etc.).

Las metas, condiciones de estudio (individual/grupal, lugar adecuado para estudiar, horarios de estudio, etc.) e implicancias en el estudio (asistencia a clases, responsabilidad, esfuerzo, participación en clases, etc.) se relacionan positivamente con los rendimientos académicos en todas las asignaturas estudiadas. Sin embargo, igual que en los estilos de aprendizaje, los coeficientes de determinación son bajos, no superando el 8\%. Estos resultados son similares a los reportado por Aravena, et al. (2017) en estudiantes de secundaria de 14 a 19 años de Santiago de Chile.

Las limitaciones del presente estudio son la imposibilidad de generar una razón concluyente de porque existen relaciones entre algunas de las variables estudiadas y que la muestra de estudiantes proviene de la misma ciudad, siendo imposible generalizar estos hallazgos. Entre las fortalezas de la investigación se encuentra el gran número de variables analizadas, entregando una gran cantidad de información, tanto descriptiva de cada variable, como del comportamiento de las relaciones entre ellas.

\section{Conclusiones}

Existe una incidencia muy baja de la antropometría, la práctica de ejercicio físico, algunos estilos de aprendizaje, de las metas, condiciones de estudio e implicancias del estudio sobre el rendimiento académico de los estudiantes de la muestra, no superando el 8\%. Estos resultados permiten asumir una independencia de las variables estudiadas sobre las 
notas que obtienen los estudiantes.

Se necesitan futuras investigaciones que abarquen diversas escuelas, regiones, niveles socioeconómicos, sexo de la muestra, etc. de nuestro país, de manera de establecer si esta situación es una característica aislada de la muestra de varones seleccionada y existen variabilidades según características socio-demográficas, o es una tendencia en estudiantes de nuestro país.

\section{Referencias}

Acevedo, V. \& Martínez, S. (2011). Relación entre estilos de aprendizaje y rendimiento académico en estudiantes de psicología e ingeniería civil. Estilos de Aprendizagem na Atualidade, 1, 1-10.

Aguirre, M., Castro, M. \& Adasme A. (2009) Factores que Inciden en el Rendimiento Escolar en Chile. Centro de Estudios de Opinión Ciudadana, 2(4), 1-4.

Alonso, C. (1991). Estilos de aprendizaje: Análisis y diagnóstico en estudiantes universitarios. Tesis doctoral, Facultad de Filosofía y Ciencias de la Educación. Universidad Complutense de Madrid, España.

Alonso, C., Gallego D. \& Honey, J. (1994). Los estilos de aprendizaje: procedimientos de diagnóstico y mejora. Bilbao: Ediciones Mensajero.

Álvarez, C. \& Albuerne, F. (2001). Rendimiento académico y estilo de aprendizaje en alumnos de segundo de bachillerato LOGSE. Aula Abierta, 77, 77-85.

Antolín, R. (2013). Motivación y rendimiento escolar en educación primaria. Tesis de Magíster, Universidad de Almería, España.

Aravena, C., Maureira, F., Flores, E. \& González, P. (2017). Incidencia del coeficiente intelectual, estilos de aprendizaje, motivos, actitudes y estrategias para el estudio sobre el rendimiento académico de los estudiantes de un colegio de Santiago. Foro Educacional, 29, 119-132. DOI: https://doi.org/10.29344/07180772.29.784

Aravena, C., Maureira, F., Flores, E., Lourido, P. \& Véliz, C. (2017). Independencia de los estilos de aprendizaje evaluados con el CHAEA-36 y el coeficiente intelectual en una muestra de estudiantes de secundaria de Chile. Revista de Psicología Iztacala, 20(4), 1451-1462.

Belaunde, I. (1994). Hábitos de estudio. Revista de la Facultad de Psicología de la Universidad Femenina del Sagrado Corazón, 2(2) ,15-18.

Bernstein, J. (1997). Inventario de hábitos de estudio de Gilbert Wrenn. México DF: Paidós.

Blumen, S., Rivero, C. \& Guerrero, D. (2011). Estilos de aprendizaje y rendimiento académico en estudiantes universitarios de educación a distancia. Revista Estilos de Aprendizaje, 7(7), 93-108.

Boza, A. \& Toscano, M. (2012) Motivo, actitudes y estrategias de aprendizaje: motivado en alumnos universitarios. Profesorado, 16(1), 125-142.

Camarero, F., Martín del Buey, F. \& Herrero, J. (2000). Estilos y estrategias de aprendizaje en estudiantes universitarios. Psicothema, 12(4), 615-622.

Cano, E. (2008). La evaluación por competencias en la educación superior. Profesorado. Revista de Currículum y Formación del Profesorado, 12(3), 1-16.
Cartagena (2008). Relación entre autoeficacia y rendimiento escolar y hábitos de estudio en alumnos de secundaria. Revista Iberoamericana sobre Calidad y Cambio en Educación, 6(3), 60-99.

Castelli, D., Hillman, C., Buck, S. \& Erwin, H. (2007). Physical fitness and academic achievement in third-and fifth-grade students. Journal of Sport and Exercise Psychology, 29(2), 239-252. DOI: https://doi.org/10.1123/jsep.29.2.239

Cazau, P. (2004). Estilos de aprendizaje: Generalidades. Recuperado de http://www.rmm.cl/biblio/doc/ 200411291631190ESTILOS\%20DE\%20APRENDVAE_Genealidadesdbc.

Chocontá, Z. (2016). Estudio relacional: motivación y rendimiento académico en ciclo cinco. Bogotá: Milla.

Edel, R. (2003). El rendimiento académico: concepto, investigación y desarrollo. REICE, 1(2), 1-15.

Gallego, D. \& Nevot, A. (2007). Los estilos de aprendizaje y la enseñanza de las matemáticas. Revista Complutense de Educación, 19(1), 95-112.

Gami, A., Witt, B., Howard, D., Erwin, P., Gami, L., Somers, V., et al. (2007). Metabolic syndrome and risk of incident cardiovascular events and death: a systematic review and meta-analysis of longitudinal studies. Journal of the American College of Cardiology, 49(4), 403-14. DOI: https://doi.org/10.1016/j.jacc.2006.09.032

Giraldo, A., Uscátegui, R., Restrepo, M. \& Estrada, A. (2012). Variabilidad del peso, la estatura y el índice de masa corporal según desarrollo puberal y tipo de colegio en adolescentes de Medellín, Colombia. Revista Chilena de Nutrición, 39(4), 136-142. DOI: http://dx.doi.org/10.4067/ S0717-75182012000400005.

Godoy, A., Valdés, P., Fariña, C., Cárcamo, F., Medina, B., Meneses, E., et al. (2015). Asociación entre la condición física, estado nutricional y rendimiento académico en estudiantes de educación física. Nutrición Hospitalaria, 32(4), 1722-1728. DOI: http://dx.doi.org/10.3305/ nh.2015.32.4.9592.

González, E. (2015). Factores de contexto socioeconómicos y educativos en estudiantes de nivel superior, sugerencia para una realidad actual. Revista Interamericana de Psicología, 49(1), 399-412.

González, L. \& González, J. (2005). Relación entre los estilos de aprendizaje, el rendimiento en matemáticas y la elección de asignaturas optativas en alumnos de ESO. Revista Electrónica de Investigación y Evaluación Educativa, 11(2), 147-165.

Haapala, E., Lintu, N., Eloranta, A., Venäläinen, T., Poikkeus, A., Ahonen, T. et al. (2018). Mediating effects of motor performance, cardiorespiratory fitness, physical activity, and sedentary behaviour on the associations of adiposity and other cardiometabolic risk factors with academic achievement in children. Journal of Sports Sciences, 36, 1-8. DOI: https://doi.org/10.1080/02640414.2018.1449562 Haapala, E., Väistö, J., Lintu, N., Westgate, K., Ekelund, U., Poikkeus, A., et al. (2017). Physical activity and sedentary time in relation to academic achievement in children. Journal of Science and Medicine in Sport, 20(6), 583589. DOI: https://doi.org/10.1016/j.jsams.2016.11.003

Hernández, F. \& Hervás, R. (2005). Enfoques y estilos de aprendizaje en Educación Superior. Revista Española de Orientación y Psicología, 16(2), 283-299. 
Honey, P. \& Munford, A. (1992). The manual of learning style. Maidenhead: Peter Honey.

Ishihara, T., Morita, N., Nakajima, T., Okita, K., Yamatsu, K. \& Sagawa, M. (2018). Direct and indirect relationships of physical fitness, weight status, and learning duration to academic performance in Japanese schoolchildren. European Journal of Sport Science, 18(2), 286-294. DOI: https://doi.org/10.1080/17461391.2017.1409273

Jiménez, M.(2000). Competencia social: intervención preventiva en la escuela. Infancia y Sociedad, 24, 21-48.

Juárez, C., Rodríguez, G. \& Escoto, M.(2012). Estudio comparativo de las estrategias de aprendizaje en tres niveles educativos. El caso de México. Trabajo presentado en el II Congreso Internacional de Psicología y Educación, Ciudad de Panamá, Panamá.

Kao, S., Westfall, D., Parks,A., Pontifex, M. \& Hillman, C. (2017). Muscular and aerobic fitness, working memory, and academic achievement in children. Medicine \& Science in Sports \& Exercise, 49(3), 500-508. DOI: 10.1249/ MSS.0000000000001132

Keefe, J. (1988). Aprendiendo perfiles de aprendizaje. La Habana: Asociación Nacional de Escuelas secundarias.

Kohler, J. (2013). Rendimiento académico, habilidades intelectuales y estrategias de aprendizaje en universitarios de lima. LIBERABIT, 19(2), 277-288.

Maquilón, J. \& Hernández, F. (2011). Influencia de la motivación en el rendimiento académico de los estudiantes de formación profesional. REIFOP, 14(1), 81-100.

Maureira, F. (2018). Principios de neuroeducación física. Madrid: Bubok Publishing.

Maureira, F., Bahamondes, V. \& Aravena, C. (2015). Relación entre los estilos de aprendizaje y el rendimiento académico de estudiantes de educación física de la USEK de Chile. Revista Ciencias de la Actividad Física UCM, 16(2), 29-36.

Maureira, F., Bravo, P., Aguilera, N., Bahamondes, V. \& Véliz, C. (2019). Relación de la composición corporal, las cualidades físicas y funciones cognitivas en estudiantes de educación física. Retos, 36, 103-106.

Maureira, F., Díaz, I., Foss, P., Ibañez, C., Molina, D., Aravena, F., et al. (2014). Relación de la práctica de actividad física y el rendimiento académico en escolares de Santiago de Chile. Revista Ciencias de la Actividad Física UCM, 15(1), 43-50.

Ministerio del Deporte (2006). Encuesta Nacional de Hábitos de Actividad Física y Deportes en la Población de 18 años y más. Recuperado de http://www.mindep.cl/wp-content/ uploads/2016/07/INFORME-FINAL-ENCUESTA-DEPORTES-COMPLETO_.pdf

Montero, E., Sepúlveda, M. \& Contreras, E. (2011). Estudio transversal de los estilos de aprendizaje y rendimiento académico en alumnos de $1^{\circ}$ año de la carrera de medicina veterinaria. Revista Estilos de Aprendizaje, 7(7), 151-159.

Oliveira, T., Pizarro,A., Costa, M., Fernandes, L., Silva, G., Mota, J., et al. (2017). Cardiorespiratory fitness, but not physical activity, is associated with academic achievement in children and adolescents. Annals of Human Biology, 44(4), 309-315. DOI: https://doi.org/10.1080/03014460.2017.1308010

Ossa, C. \& Lagos, N. (2013). Estilos de aprendizaje y rendimiento académico en estudiantes de Pedagogía de Educación General Básica (primaria) de una universidad pública en Chile. Revista Estilos de Aprendizaje, 11(11), 1-12.

Owen, K., Parker, P., Astell, T. \& Lonsdale, C. (2018). Effects of physical activity and breaks on mathematics engagement in adolescents. Journal of Science and Medicine in Sport, 21(1),63-68.DOI:https://doi.org/10.1016/j.jsams.2017.07.002

Palou, P., Ponseti, X., Gili, M., Borras, P. \& Vidal, J. (2005). Motivos para el inicio, mantenimiento y abandono de la práctica deportiva en los preadolescentes de la isla de Mallorca. Apunts, Educación Física y Deportes, 81, 5-11.

Pellicer, M., García, X., Morales, J., Serra, P., Solana, M., González, L., etal.(2015). Physical activity, physical fitness and academic achievement in adolescents: a selforganizing maps approach. Health Education Research, 30(3), 436-448. DOI: https:// doi.org/10.1093/her/cyv016

Pinel, C., Chacón, R., Castro, M., Espejo, T., Zurita, F. \& Péez,A. (2017). Diferencias de género en relación con el Índice de Masa Corporal, calidad de la dieta y actividades sedentarias en niños de 10 a 12 años. Retos, 31, 176-80.

Pintrich, P. \& Schunk, D. (2006). Motivación en contextos educativos. Madrid: Pearson.

Pizarro, R. \& Clark, S. (1998). Currículo del hogar y aprendizajes educativos. Interacción versus estatus. Revista de Psicología de la Universidad de Chile, 7, 25-33.

Prieto, J. \& Martínez, C. (2016). Lapráctica de actividad física y su relación con el rendimiento académico. Revista de Educación Física: Renovar la Teoría y Práctica, 144, 3-12.

Quintanal, F. (2011). Influencia de los estilos de aprendizaje en el rendimiento escolar de física y química de secundaria. Tesis de doctorado, UNED, España.

Reed, J., Einstein, G, Hahn, E., Hooker, S., Gross, V. \& Kravitz, J. (2010). Examining the impact of integrating physical activity on fluid intelligence and academic performance in an elementary school setting: a preliminary investigation. Journal of Physical Activity and Health, 7(3), 343-351.

Romero, C. (2006). El síndromemetabólico. Rev Med Urug, 22(3), $108-21$.

Ruiz, B., Trillos, J. \& Morales, J. (2006). Estilos de aprendizaje y rendimiento académico en estudiantes universitarios. Revista Galego-Portuguesa de Psicoloxia e Educación, 13(1112), 441-457.

Serra-Olivares, J., Muñoz, C., Cejudo, C. \& Gil, P.(2017). Estilos de aprendizaje y rendimiento académico de universitarios de Educación Física chilenos. Retos, 32, 62-67.

Suazo, I. (2007). Estilos de Aprendizaje y su correlación con el rendimiento académico en anatomía humana normal. International Journal of Morphology, 25(2), 367-373. DOI: http://dx.doi.org/10.4067/S0717-95022007000200022

Tobarra, E., Castro, O. \& Badilla, R. (2015). Estado nutricional y características socioepidemiológicas de escolares chilenos, OMS 2007. Revista Chilena de Pediatría, 86(1), 12-17.DOI: http://dx.doi.org/10.1016/j.rchipe.2015.04.003.

Tonconi, Q. (2010). Factores que influyen en el rendimiento académico y la deserción de los estudiantes de la Facultad de Ingeniería Económica de la UNA-Puno (Perú). Recuperado de http://www.eumed.net/rev/ced/11/jtq.htm

Van Dijk, M., De Groot, R., Savelberg, H., Van Acker, F. \& Kirschner, P. (2014). The association between objectively measured physical activity and academic achievement in Dutch adolescents: findings from the GOALS study. Journal of Sport and Exercise Psychology, 36(5), 460-473. DOI: https:/ /doi.org/10.1123/jsep.2014-0014

Wilmore, J. \& Costill, D. (2007). Fisiología del esfuerzo físico yel deporte. Madrid: Paidotribo. 